\title{
Evaluation of the antioxidant enzyme activity level in patients with alopecia areata
}

\author{
Anna Cwynar ${ }^{1}$, Dorota Olszewska-Słonina ${ }^{1}$, Rafał Czajkowski², Elżbieta Piskorska ${ }^{1}$, Iga Hołyńska-Iwan ${ }^{1}$, \\ Piotr Kaczorowski ${ }^{1}$, Magdalena Lampka ${ }^{1}$
}

'Department of Pathobiochemistry and Clinical Chemistry, Collegium Medicum in Bydgoszcz, Nicolaus Copernicus University in Torun, Poland

${ }^{2}$ Department of Dermatology, Sexually Transmitted Disorders and Immunodermatology, Collegium Medicum in Bydgoszcz, Nicolaus Copernicus University in Torun, Poland

Adv Dermatol Allergol 2018; XXXV (4): 423-424

DOI: https://doi.org/10.5114/ada.2018.77674

Alopecia areata (AA) is a disease with complex and not fully known etiopathogenesis, associated with hair follicle damage [1]. The disease affects only hair containing melanin, therefore grey streaks remain in the foci of alopecia. It happens that after 3 to 6 months there is spontaneous regrowth - new hair is then free of dye and their repeat pigmentation occurs only after a few weeks [2].

One of the theories that elucidates the pathomechanism of AA is the theory of oxidative stress [3]. The proof of the validity of this theory can be the presence of new grey hair in patients with AA. The study has shown that millimolar concentrations of hydrogen peroxide $\left(\mathrm{H}_{2} \mathrm{O}_{2}\right)$ are accumulated within grey hair [4]. Its presence inhibits the conversion of tyrosine into melanin by inactivation of the enzyme tyrosinase. The increase in the level of $\mathrm{H}_{2} \mathrm{O}_{2}$ is most probably due to a decrease in activity of catalase (CAT) responsible for its detoxification [5].

$\mathrm{H}_{2} \mathrm{O}_{2}$ decomposition is assumed to occur mainly with the participation of glutathione peroxidase (GPx), thus CAT acts only as an auxiliary and is active when extremely high concentrations of $\mathrm{H}_{2} \mathrm{O}_{2}$ occur [6]. In turn, the biological role of superoxide dismutase (SOD) is to catalyse reactions of dismutation of superoxide radicals with simultaneous generation of $\mathrm{H}_{2} \mathrm{O}_{2}$ [7]. In physiological conditions these enzymes operate together; for this reason, the inactivation of any of these enzymes will weaken the antioxidant defence of the body.

The objective of this study was to evaluate the state of oxidant-antioxidant balance in patients with AA by assessment of the most important antioxidant enzymes, GPX, CAT and SOD, in patients with AA. The study included 30 AA patients (23 females/8 males, mean age: 34.4 $\pm 10.9)$ and the control group consisting of 30 age- and sex-matched healthy volunteers (17 females/13 males, mean age: $37.3 \pm 17.4$ ). There was no significant difference between groups with regard to age and body mass index (BMI).

A disturbance in the oxidative-antioxidant balance was demonstrated by a statistically significant decrease in the GPx activity level in blood erythrocytes samples from the patients with AA as compared to the control group ( $p=0.001)$. Also a decrease in CAT activity was noted in patients with AA as compared to the control group, but these differences were not statistically significant ( $p=0.491)$. A slight, statistically insignificant increase was observed in the activity of SOD in patients with AA as compared to the control ( $p=0.221)$ (Table 1).

Oxidative stress is a physiological consequence of the aerobic metabolism of cells. However, in some cases its intensity exceeds the adaptability of the body, which in consequence may lead to development of diseases with free radical background. Enzymatic antioxidative mechanisms neutralizing reactive oxygen species (ROS) should form a consistent and efficient antioxidant defence system [8]. For this purpose, the activity of the most important antioxidant enzymes, GPx, CAT and SOD, was evaluated in patients with AA.

Glutathione peroxidase is involved in the reduction of hydrogen peroxide and organic hydroperoxides. In addition to the protective function towards the cell membranes (it protects against their peroxidation damage), it also controls the proper course of cell metabolism. The activity of glutathione peroxidase changes in disorders occurring in the body as a result of oxidative stress $[3,6]$. Substantial reduction in this enzyme observed in alope-

Address for correspondence: Anna Cwynar, Department of Pathobiochemistry and Clinical Chemistry, Collegium Medicum in Bydgoszcz, Nicolaus Copernicus University, 9 M. Curie-Skłodowskiej St, 85-094 Bydgoszcz Poland, phone: +48 793414790

e-mail: anna.cwynar@vp.pl

Received: 18.07.2017, accepted: 4.08.2017. 
Table 1. Activity of GPx, CAT and SOD in peripheral blood erythrocytes of alopecia patients and healthy subject

\begin{tabular}{lccc}
\hline Parameter & AA patients $(n=30)$ & Healthy subjects $(n=30)$ & $P$-value \\
\hline GPx $[\mathrm{U} / \mathrm{gHb}]$ & $7.56 \pm 3.32$ & $11.56 \pm 6.03$ & 0.001 \\
\hline $\mathrm{CAT}[\mathrm{IU} / \mathrm{gHb}]$ & $57.73 \pm 13.65$ & $64.21 \pm 10.73$ & 0.491 \\
\hline SOD $[\mathrm{U} / \mathrm{gHb}]$ & $699.39 \pm 81.74$ & $674.66 \pm 69.84$ & 0.221 \\
\hline
\end{tabular}

GPx-glutathione peroxidase, CAT - catalase, SOD - superoxide dismutase, $p$-statistical significance.

cia areata may suggest an oxidative-antioxidative balance disorder associated with this condition. Moreover, it may also affect the presence of elevated hydrogen peroxide concentrations in the course of AA. The observed decrease in CAT activity and higher SOD activity in AA patients may further exacerbate $\mathrm{H}_{2} \mathrm{O}_{2}$ concentration within the hair follicle. The results obtained may indicate the ineffectiveness of enzymatic antioxidant defence systems in AA, and even explain the cause of appearance of grey hair in this group of patients. So far, many observations have shown that the process underlying greying is oxidative stress induced by hydrogen peroxide.

Laboratory evaluation of the activity of individual antioxidant enzymes provides valuable information on the condition of the enzymatic antioxidant barrier in AA. However, a wider use of the ability to determine the activity of these enzymes, both for diagnostic-prognostic and prophylactic purposes, remains a matter of the future.

\section{Acknowledgments}

The authors thank the Department of Medical Biology (Nicolaus Copernicus University in Torun) for technical support during the preparation of this manuscript.

\section{Conflict of interest}

The authors declare no conflict of interest.

\section{References}

1. McElwee KJ, Gilhar A, Tobin DJ, et al. What causes alopecia areata? Exp Dermatol 2013; 22: 609-26.

2. Jia WX, Mao QX, Xiao XM, et al. Patchy alopecia areata sparing gray hairs: a case series. Postep Dermatol Alergol 2014; 31: 113-6.

3. Prie BE, Voiculescu VM, Ionescu-Bozdog OB, et al. Oxidative stress and alopecia areata. J Med Life 2015; 8: 43-6.

4. Trüeb RM. Oxidative stress in ageing of hair. Int I Trichology 2009; 1: 6-14.

5. Wood JM, Decker H, Hartmann H, et al. Senile hair graying: $\mathrm{H}_{2} \mathrm{O}_{2}$-mediated oxidative stress affects human hair color by blunting methionine sulfoxide repair. FASEB J 2009; 23: 2065-75.

6. Day BJ. Catalase and glutathione peroxidase mimics. Biochem Pharmacol 2009; 77: 285-96.

7. Fridovich I. Superoxide anion radical $\left(\mathrm{O}_{2}^{-}\right)^{-}$, superoxide dismutases, and related matters. J Biol Chem 1997; 272: 18515-7.

8. Lobo V, Patil A, Phatak A, et al. Free radicals, antioxidants and functional foods: impact on human health. Pharmacogn Rev 2010; 4: 118-26. 